\title{
Domain differences in absolute judgments of category membership: Evidence for an essentialist account of categorization
}

\author{
GIL DIESENDRUCK \\ University of Arizona, Tucson, Arizona \\ and \\ SUSAN A. GELMAN \\ University of Michigan, Ann Arbor, Michigan
}

\begin{abstract}
There has been some debate about the correspondence between typicality gradients and category membership. The present study investigates the relationship between these two measures in the domains of animals and artifacts. Forty-two adults judged the degree of typicality or category membership of 293 animals and artifacts. The subjects' tendency for animals, but not for artifacts, was to make more absolute ratings on category membership (i.e., judging exemplars as definitely members or definitely not members of their respective category) than on typicality. More importantly, at almost every level of typicality, subjects were more likely to make absolute judgments of category membership for animals than for artifacts. These results indicate that people treat category membership of animals as relatively absolute (which best fits an essentialist model of categorization) and treat category membership of artifacts as relatively graded (which best fits a prototype model of categorization). These domain differences add crucial supporting evidence for claims about the domain-specificity of essentialism.
\end{abstract}

For the last two decades, a considerable amount of research in categorization has been devoted to understanding the relationship between typicality and category structure. Rosch $(1975,1978)$ postulated that category boundaries were defined by similarity relations to a prototype, and, thus, typicality judgments provided reliable assessments of category structure. In the 1980 s, a number of researchers challenged this latter conclusion by finding graded judgments of typicality even for categories in which membership is all-or-none (e.g., odd numbers; Armstrong, Gleitman, \& Gleitman, 1983). These findings pointed to the importance of assessing the membership structure of categories independently of their typicality structure and, more importantly, appraising the relationship between judgments of typicality and of category membership. The present study addressed this last question, specifically focusing on whether the nature of this relationship differs between domains.

According to a prototype account, the nature of the relationship between typicality and category membership is still basically as Rosch (1978) advocated: Considerations of typicality underlie decisions about category mem-

The first author was supported by a grant from the Flinn Foundation of Phoenix to the University of Arizona for the support of Cognitive Neuroscience. The authors thank Marla Stobinsky for help collecting and coding the data. Correspondence should be addressed to G. Diesendruck, who is now at the Department of Psychology, Bar Ilan University, Ramat Gan 52900, Israel (e-mail: dieseng@ mail.biu.ac.il). bership. Similarity to a prototype reflects not only how good an example of a category an instance is but also how clearly a member of that category the instance is (Hampton, 1995). Thus, even if we measure these two types of judgments independently, prototype models predict a monotonic relation between typicality ratings and judgments of category membership (Hampton, 1998). That is, as the similarity of an exemplar to the prototype of a category decreases, so does the probability of categorizing the exemplar as a member of the category. Nonetheless, given the fuzziness of category boundaries, very atypical items might still have a small but nonzero probability of being considered members of the category by some people and yet have a slowly increasing probability of being considered definitely not members of the target category by other people (see Hampton, 1995, for a graphic instantiation of a prototype model).

The main alternative to similarity-based models, such as prototypes, has been one that emphasizes the role of theories in conceptual organization (Murphy \& Medin, 1985). One corollary of this approach is the essentialist account of categorization (Medin, 1989). According to this view, people have a belief that categories in certain domains have nonobvious essential properties that determine what members of these categories are. People do not necessarily know what the essential properties of a category are, but rather they simply have a belief that these properties exist. On this account, all members of a category are believed to possess the category's essential properties to the same degree and are therefore considered members of the category to the 
same extent. Members of a category may differ, however, in the typicality of their nonessential features (e.g., physical appearance) and therefore may vary in how good an example of the category they are. The essentialist account, then, attempts to capture the intuition that, for instance, although a Chihuahua and a German shepherd differ in how representative they are of the category $d o g$, the former is as much a dog as the latter. More generally, the essentialist account argues that categorization is all-or-none: Items are judged absolutely as either members of their category or not members of their category.

A number of studies suggest that animals may be relatively more essentialized than artifacts. Preschoolers draw more powerful inductive inferences from animal categories than from artifact categories (Gelman, 1988; Gelman \& Markman, 1986); they believe that internal properties define the kind of animal something is, but they do not believe that functional properties define the kind of artifact something is (Diesendruck, Gelman, \& Lebowitz, 1998); and they seem more likely to treat artifactual categories as invented conventions than as objective natural groupings (Kalish, 1998). Studies with adults reveal that extreme transformations in an animal's superficial features (e.g., a change in the color of its fur or smell) do not alter how they classify an animal, though comparable transformations in an artifact may change its membership (Rips, 1989; see also Keil, 1989, for similar findings with school-aged children). Studies with adults also reveal that, in general, adults believe animal categories have defining features to a greater extent than artifact categories do (Malt, 1990; Malt \& Johnson, 1992). Similar domain differences have also been argued for in linguistics (Wierzbicka, 1984), philosophy (Schwartz, 1978), and anthropology (Atran, 1995).

The implication of these studies for the present question is that if indeed people's conception of animals is characterized by essentialist beliefs, whereas people's conception of artifacts is not, then we should expect people to judge the category membership of animals, but not of artifacts, in an absolute manner independent of typicality. For instance, people should be more likely to judge a penguin as definitely a bird than they are to judge a beanbag as definitely a chair (assuming a penguin is as atypical a bird as a beanbag is an atypical chair); and they should be more likely to judge a bat as definitely not a bird than they are to judge an ottoman as definitely not a chair (again assuming a bat is as atypical a bird as an ottoman is an atypical chair). The alternative prototype-based hypothesis is that judgments of typicality and category membership should be equally graded in both domains.

Three papers provided data with some bearing on these hypotheses. Barr and Caplan (1987) collected typicality and category membership ratings on 13 different categories from two groups of adults. Although their analyses did not focus on domain differences, their data revealed differences between animals and artifacts com- patible with the essentialist prediction described above. First, the two categories with the highest proportion of absolute judgments of category membership were the two animal categories used in their study: bird and mammal $(81 \%$ of the subjects, relative to $70 \%$ for the artifact categories clothing, furniture, tools, toys, vehicles, and weapons; see Barr \& Caplan's, 1987, Table 3, p. 407). Second, the proportion of absolute responses in typicality and membership ratings differed the most for the two animal categories (see Barr \& Caplan's, 1987, Table 7, p. 410).

A second study also supplied results in the direction predicted by the essentialist account. McCloskey and Glucksberg (1978) presented adults with pairs consisting of a candidate exemplar (e.g., robin) and a category name (e.g., bird). One group of adults was asked to rate the typicality of the exemplars on a 10-point scale. On two separate occasions ( 1 month apart), a second group of adults was asked to decide whether or not each exemplar was a member of the category. The authors did not analyze the data with respect to domains; however, as revealed in their Appendix (McCloskey \& Glucksberg, 1978 , pp. 467-471), for items with an intermediate level of typicality (i.e., ratings of 4.00-6.99), there were fewer between-subjects disagreements and within-subjects inconsistencies about the category membership of animals than of artifacts. In other words, there was a tendency for the membership status of animals to be less ambiguous than the membership status of artifacts.

However, neither of these two studies directly tested for domain differences in the relationship between membership and typicality. In an attempt to do so, Hampton (1998) reanalyzed McCloskey and Glucksberg's (1978) data. He found that, for both animals and artifacts, there was a monotonic relationship between typicality and the probability of category membership, a finding he interpreted as supporting a prototype account. Nonetheless, he also found that, for animals, but not for artifacts, similarity in core features was a stronger predictor of positive categorization than similarity in superficial features, a finding consistent with an essentialist account. In general, however, the design of the original study was not optimal to test the essentialist claim about category membership (nor was it designed to do so). Mainly, the problem arises from the fact that subjects had to make a forced choice as to whether something was or was not of kind $X$. With this paradigm, it is impossible to establish whether a positive categorization implies that the subject believes an item is absolutely a member of category $X$ or instead believes the item to be more a member of category $X$ than not. In other words, one cannot determine the absoluteness (and conversely the gradedness) of categorization with this method. In this light, the finding of a monotonic relation between typicality and category membership is consistent not only with a prototype account but also with an essentialist account. The essentialist interpretation would be that, as typicality increases, the 
probability of absolute positive categorization increases, but, at the same time, the probability of absolute negative categorization decreases at the same rate.

In the only direct test (to our knowledge) of the essentialist hypotheses, Kalish (1995) found no differences in adults' categorization of animals and artifacts. In his Study 1 , subjects were as likely to make absolute judgments of category membership (i.e., to say that an item is "not at all" or is "completely" a member of its category) for animals ( $38 \%$ ) as they were for artifacts ( $41 \%$ ); altogether, ratings of category membership for both animals and artifacts were graded. As Kalish (1995) noted, however, a few aspects of his procedure might have influenced the results. First, a small number of items was used for each category (e.g., three or four for the three animal categories), only a limited set of categories was used in each domain (two artifact and three animal categories), and both artifact categories were at the superordinate level, whereas only one of the animal categories was at that level. These aspects of the stimulus set raise doubts about the generalizability of the findings to the entire domains. Second, the items selected (primarily for artifacts and animals) were intended to be atypical instances of their respective categories, but no independent measure was used to verify or control for their typicality. In fact, on closer inspection, most of the animal items were hybrid rather than atypical instances (e.g., the item mule was intended to be an atypical horse, but, in fact, is the hybrid product of a horse and a donkey). Consequently, as Kalish (1995) pointed out, it is possible that a subject's graded judgment of an animal's category membership reflected uncertainty regarding the membership of that animal rather than certainty that the animal was an intermediate member of a category. Finally, all subjects were first asked to provide typicality ratings and then category membership ratings. It is possible, then, that the initial gradedness of subjects' typicality judgments biased their subsequent judgments of category membership.

Following his review of this literature, Hampton (1998) concludes that

None of the studies so far described have adopted the most direct way to test the monotonicity between similarity and category membership. This would be to obtain measures of category membership and typicality from independent groups of participants and to test the monotonicity of the results directly. (p. 145)

For reasons we pointed out earlier, we believe Hampton's (1998) analysis of the McCloskey and Glucksberg (1978) data also did not provide an ideal test of the relationship between typicality and category membership. In the present study, we attempted to do so by addressing some of the methodological issues raised above regarding previous studies. First, instead of using a dichotomy, as McCloskey and Glucksberg did, category membership was assessed on a 7-point scale so as to better capture gradedness in responses. Second, relative to Kalish (1995), subjects in the present study were asked to provide ratings of a larger set of instances in each category (between 16 and 24) and ratings of a larger number of categories in each domain, at both basic and superordinate levels. We believe this modification would increase the representativeness of our results. Third, the instances in each category represented a wider range of typicality relative to Kalish's (1995) stimulus set and thus allowed an analysis of domain differences in category membership across the entire range of typicality. Finally, type of rating (i.e., typicality or category membership) was a betweensubjects factor, as called for by Hampton (1998) and in contrast to Kalish's (1995) within-subjects design. This was done so as to (1) enable the use of typicality judgments as an independent measure by which to evaluate the distribution of absolute category membership ratings across the spectrum of typicality and (2) rule out having subjects biased by the typicality ratings when asked for judgments of category membership.

The alternative hypotheses are that, if indeed people reason about animals in an essentialist way to a greater extent than they do about artifacts, then (1) for animals, subjects should give more absolute judgments for category membership than for typicality, but, for artifacts, subjects' typicality and membership ratings should be equally graded, and (2) in general, controlling for typicality, there should be more absolute membership ratings for animals than for artifacts. According to the prototype account, on the other hand, membership and typicality responses should be related and equally graded for both animals and artifacts.

\section{METHOD}

\section{Subjects}

Forty-two undergraduate students from the University of Arizona participated in this study. The students received course credit for their participation. Half of the subjects provided ratings of typicality, and the other half provided judgments of category membership.

\section{Stimuli}

There were 14 categories of items: 8 categories of artifacts, and 6 categories of animals. The categories were at both the superordinate level (e.g., furniture, insect) and the basic level (e.g., car, dog). The categories selected were categories that have been commonly used in studies of typicality and category membership.

A list of items varying in their typicality was chosen for each category. Findings from earlier studies on typicality were used to help generate the list (Barr \& Caplan, 1987; Battig \& Montague, 1969; Rosch, 1975). The resulting list of items for each category ranged from typical members of the category (e.g., sparrow for the category bird) to atypical members (e.g., penguin) to nonmembers (e.g., bat). On average, there were 21 exemplars per category (range $=$ 16-24), for a total of 293 items. The complete list of categories and items is presented in the Appendix.

\section{Procedure}

The subjects were randomly assigned to provide ratings of either the typicality or the category membership of all 293 items. Each subject was given a rating questionnaire to fill out. The first page 
of the questionnaire contained general instructions about the type of rating the subject was to provide. To make sure that the subjects understood their task, the experimenter read the first page out loud. The remaining 14 pages of the questionnaire had the same basic format. On the very top of each page, there was the name of a category (e.g., furniture), and below it were the names of items (e.g., desk, sofa, lamp, clock) to be rated in terms of their typicality or membership in that category. The subjects were asked to write down their rating in a space next to the name of each item. The 14 categories appeared in random order across subjects. There were two quasi-random orders of items for each category. The only constraint on the order of appearance of the items was that the first item in each category was always a typical member of the category (e.g., the item chair for the category furniture).

For typicality ratings, the procedure was similar to that developed by Rosch (1975). The subjects were asked to judge "how good an example" of the category each item was. Ratings were made on the basis of a 7-point scale, in which 1 stood for very good example and 7 stood for very poor example. For membership ratings, the subjects were asked to judge "how clearly a member" of the category each item was. Again, ratings were made on the basis of a 7-point scale, in which 1 stood for definitely a member and 7 stood for definitely not a member. The extremes of the membership scale ( 1 or 7) thus constituted "absolute" judgments of category membership. For both ratings, the subjects were instructed to use the numbers between 1 and 7 to indicate intermediate degrees of typicality or category membership.

\section{RESULTS}

Our first analysis examined the proportion of items receiving extreme ( 1 or 7$)$ ratings of typicality and category membership across domains. A series of analyses were then conducted to address directly Hypotheses 1 and 2 outlined in the introduction. Hypothesis 1 was addressed by analyzing differences between judgment types in each domain separately. We accomplished this by looking at differences between judgment types in the proportion of absolute ratings and by analyzing the distribution of the subjects consistently making absolute ratings. To address Hypothesis 2, we conducted two analyses. First, we controlled for differences in typicality between the domains and then analyzed the differences in the proportion of items in each domain receiving absolute membership ratings. Second, we derived regression lines on the proportion of subjects making absolute membership ratings as a function of mean typicality.

For the first analysis, we conducted an analysis of variance (ANOVA) on the arcsine-transformed proportion of items receiving extreme ratings. For this analysis, judgment type (typicality vs. membership) was entered as a between-subjects variable, and domain (artifacts vs. animals) was entered as a within-subjects variable. Table 1 presents the proportion of extreme typicality and membership ratings for each of the 14 categories. The effect of domain was significant $[F(1,40)=55.67, p<.0001]$. The subjects made more extreme ratings for animals than for artifacts. The effect of judgment type was not significant $[F(1,40)=3.85$, n.s. $]$, and neither was the interaction between domain and judgment type $[F(1,40)=$ 1.22, n.s.].
Table 1

Proportion of Items in Each Category Receiving Absolute (1 or 7 ) Typicality or Membership Ratings

\begin{tabular}{llc}
\hline Category $n$ Items & $\begin{array}{c}\text { Proportion of Extreme } \\
\text { Typicality Ratings }\end{array}$ & $\begin{array}{c}\text { Proportion of Extreme } \\
\text { Membership Ratings }\end{array}$ \\
\hline
\end{tabular}

\begin{tabular}{llll}
\hline \multicolumn{3}{c}{ Animals } \\
Mammal & 22 & .72 & .96 \\
Reptile & 16 & .75 & .85 \\
Insect & 23 & .73 & .82 \\
Dog & 20 & .65 & .80 \\
Fish & 22 & .66 & .65 \\
Bird & 22 & .67 & .84 \\
Total & & .70 & .82 \\
& & Artifacts & \\
Furniture & 23 & .58 & .59 \\
Clothing & 21 & .54 & .68 \\
Weapon & 22 & .55 & .59 \\
Tool & 23 & .58 & .62 \\
Vehicle & 24 & .52 & .54 \\
Chair & 16 & .43 & .46 \\
Car & 20 & .56 & .69 \\
Shoe & 19 & .53 & .68 \\
Total & & .54 & .61 \\
\hline
\end{tabular}

The essentialist Hypothesis 1 predicts that there will be more extreme membership ratings than typicality ratings for animals, but no difference between the two ratings on this measure for artifacts. To address this hypothesis directly, we conducted two analyses. First, simple-effects tests on the above ANOVA revealed no significant effect of judgment type for artifacts $[F(1,40)=$ 1.46$, n.s. $]$ but a significant effect for animals $[F(1,40)=$ $4.76, p<.05]$. A second analysis looked at the distribution of subjects in terms of consistency of extreme ratings (defined as at least $75 \%$ of the responses being 1 or 7) for each domain separately. For artifacts, only 4 of the 21 subjects providing membership judgments made extreme ratings consistently, and only 3 of the 21 providing typicality judgments did so $\left[\chi^{2}(1, N=42)=0.17\right.$, n.s. $]$. For animals, on the other hand, 18 of the 21 membership subjects gave extreme ratings consistently, but only 11 of the 21 typicality subjects did so $\left[\chi^{2}(1, N=42)=5.46\right.$, $p<.02]$. In sum, as predicted by an essentialist account, for animals, there were more extreme ratings of membership than of typicality, whereas, for artifacts, the two types of rating were equally graded. However, as can be seen in Table 1 and as indicated by the nonsignificant interaction in the ANOVA, this effect was small.

To address Hypothesis 2, it was necessary to control for typicality differences between the domains. As revealed in Table 1, there seem to be differences between the domains not only in terms of membership ratings but also in terms of typicality. The prediction, however, is that animal items will receive more absolute ratings of category membership than artifact items of equal typicality. For this purpose, we sorted all items into six intervals according to their mean typicality ratings: $1-1.9,2-2.9$, $3-3.9,4-4.9,5-5.9$, and $6-7$. We then compared the two 
Table 2

Proportion of Absolute Membership Ratings and Mean Typicality Ratings by Typicality Intervals

\begin{tabular}{|c|c|c|c|c|c|c|}
\hline \multirow{2}{*}{$\begin{array}{l}\text { Typicality } \\
\text { Interval }\end{array}$} & \multicolumn{2}{|c|}{ Proportion of Definitely a Member } & \multicolumn{2}{|c|}{ Proportion of Definitely Not a Member } & \multicolumn{2}{|c|}{ Mean Typicality Rating } \\
\hline & Animals & Artifacts & Animals & Artifacts & Animals & Artifacts \\
\hline $1-1.9$ & .92 & .91 & .01 & .01 & 1.5 & $1.6^{*}$ \\
\hline $2-2.9$ & .80 & $.61 \dagger$ & .04 & .05 & 2.3 & 2.5 \\
\hline $3-3.9$ & .55 & $.38 \dagger$ & .12 & .09 & 3.4 & 3.5 \\
\hline $4-4.9$ & .19 & .19 & .27 & $.16^{*}$ & 4.3 & 4.3 \\
\hline $5-5.9$ & .09 & .06 & .53 & $.35 \dagger$ & 5.4 & 5.1 \\
\hline $6-7$ & .01 & .03 & .86 & $.53 \dagger$ & 6.5 & 6.3 \\
\hline
\end{tabular}

Note- $F \mathrm{~s}(1,20)$ are on the arcsine-transformed proportions listed in the table. The effects refer to differences between the two domains (i.e., a difference between the two columns left of the asterisk or dagger). $\quad{ }^{*} p<.05 . \quad{ }^{\dagger} p<.005$.

domains in terms of the arcsine-transformed proportion of items in each interval that were judged definitely a member and definitely not a member. The hypothesis was that, within each interval of typicality, there would be more absolute ratings of membership for animals than for artifacts. Table 2 presents these data, together with data on the mean typicality ratings of items in each domain within each interval. Recall that independent groups of subjects provided the typicality and membership ratings.

For all but the first interval of typicality, the subjects made more absolute judgments of category membership for animal items than for artifact items. As indicated in Table 2, the subjects were more likely to rate animal items with mean typicality ratings between 2 and 2.9 as definitely members of their respective categories than artifact items with mean typicality ratings in the same interval $[F(1,20)=19.93, p<.001]$. The same domain difference was found for items in the interval 3-3.9 $[F(1,20)=21.21, p<.001]$. In fact, the subjects were as likely to judge animal items within the 3-3.9 interval as definitely members of their respective categories as they were to judge artifact items in the interval 2-2.9 (n.s.). As for absolute judgments of nonmembership, in each of the three intervals of mean typicality ratings between 4 and 7 , the subjects were more likely to judge animal items than artifact items as definitely not members of their respective categories [for $4-4.9, F(1,20)=5.03, p<.05$; for $5-5.9, F(1,20)=11.15, p<.005$; for $6-7, F(1,20)=$ $26.01, p<.001]$. In fact, the subjects were as likely to judge animal items within the 3-3.9 interval as definitely not members of their respective categories as they were artifact items in the interval 4-4.9; the same was the case for animal items in the 4-4.9 interval and artifact items in the 5-5.9, and for animal items in the 5-5.9 interval and artifact items in the 6-7 interval (all $p s>.05$ ). Finally, as revealed in Table 2 , the only statistically significant difference between domains in terms of mean typicality ratings was for the interval $1-1.9$, which was the only interval in which the two domains did not differ on either of the membership measures.

To further address Hypothesis 2 and more directly assess the models with respect to the relationship between typicality and category membership, we plotted the data for animals and artifacts on separate graphs relating the mean typicality of items and the proportion of subjects who made absolute categorization of the items. Linear regressions were then derived for each domain. According to the essentialist model, the degree of linear correlation between mean typicality and absolute category membership should be close to zero. After all, an item will be considered either definitely a member of a category or definitely not a member. Graphically, the idealized essentialist model predicts a straight line across the entire range of typicality at a constant height in which the proportion of absolute category membership equals 1 . According to a prototype model, on the other hand, there should be a nonzero correlation between the two measures. The hypothesis is that as typicality decreases, so does the probability of absolute positive categorization, and, at the fuzzy boundaries of the category, the probability of absolute negative categorization may start to rise. That is, graphically, the prototype model predicts a curve starting at a high proportion of absolute categorization (for highly typical items) and with a negative slope as typicality decreases (see Hampton, 1995). The curve may acquire a slight positive slope at extremes of atypicality, as ratings of absolute negative categorization start to occur. The alternative predictions for the nature of the linear regressions are straightforward: The essentialist model predicts a sharper slope for artifacts than for animals, whereas the prototype model predicts equal and nonzero slopes for both domains.

The analyses revealed that the slopes on both regressions were different from zero [for animals, $\beta=-.52$, $t(123)=-6.80, p<.0001$; for artifacts, $\beta=-.70$, $t(166)=-12.75, p<.0001]$. Importantly, the slope for artifacts was significantly sharper than the slope for animals $[t(289)=3.79, p<.0005]$. The intercepts were not significantly different from 1 and were not significantly different from each other. The regression lines and the scatterplots of the data are presented in Figure 1. The fact that the animal line is higher than the artifact line illustrates that there was a larger proportion of absolute category membership for animal items than for artifact items, along almost the entire continuum of typicality.

In sum, looking at both the proportion of items getting absolute categorization (Table 2) and the proportion of subjects making absolute categorization (Figure 1), the pattern is quite clear: more absolute judgments of category membership for animals than for artifacts of equal typicality. 

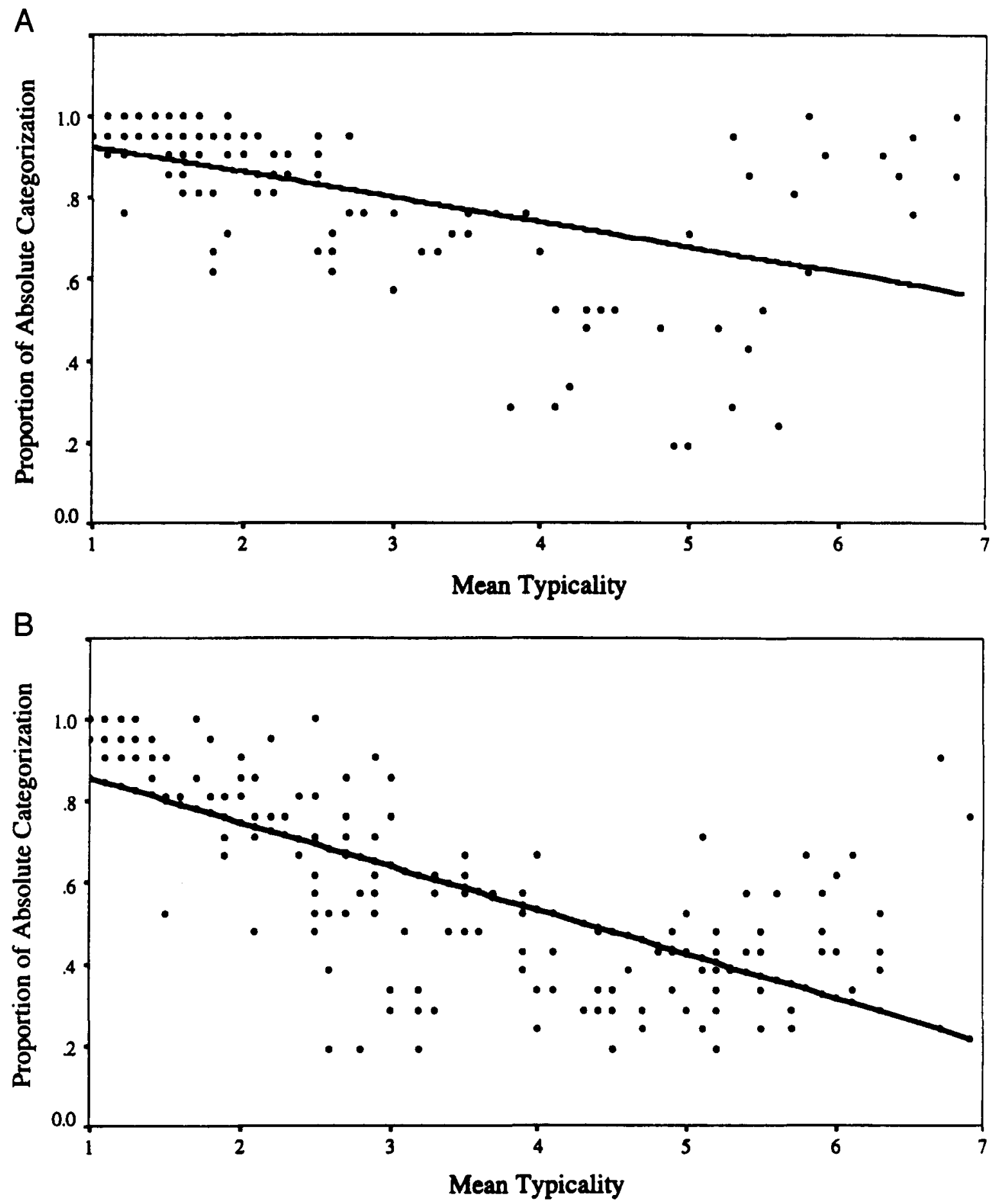

Figure 1. Scatterplot and regression lines relating proportion of subjects making absolute categorization to the mean typicality of items. (A) Animals. (B) Artifacts.

\section{DISCUSSION}

The present study investigated the extent to which adults' conception of the membership structure of animal categories is different from that of artifact categories. In doing so, this study is the first to provide a model for the relationship between gradients of typicality and gradients of category membership using natural, as opposed to artificially constructed, categories. We found that category structure is indeed importantly different across the two domains. First, the correspondence between typicality and category membership tended to be tighter for ar- 
tifacts than for animals. Second, and more importantly, the subjects were more likely to judge animal instances as definitely members or definitely not members of their respective categories than artifact instances of equal typicality. For example, among relatively atypical items (e.g., with mean typicality ratings in the $3 \mathrm{~s}$ ), the subjects rated the majority of animals as definitely members of their respective categories (55\%), and did so significantly more than for artifact items $(38 \%)$. This difference between the two domains in terms of the frequency of absolute judgments of category membership was manifest across nearly the entire range of typicality.

The present results are consistent with and strengthen the findings of other studies assessing judgments of category membership. Consistent with Barr and Caplan's (1987) data, in the present study, the subjects were more likely to make absolute judgments of category membership for animals than for artifacts, and the proportion of absolute membership and typicality responses differed most for the domain of animals. Analogous to McCloskey and Glucksberg (1978), in the present study, atypical animal exemplars were more likely to be considered unambiguously members or unambiguously not members of their categories than were atypical artifact exemplars.

The present findings, however, contrast with those of Kalish (1995). In his Study 1, no differences between the two domains were found in terms of subjects' likelihood of making absolute judgments of category membership; altogether, subjects rated only $38 \%$ of the animal items as definitely members or definitely not members of their respective categories. Two methodological differences might help explain this disparity. First, the larger number of categories and the greater range of candidate exemplars for each category used in the present study rendered a more representative sample of items in each domain than the stimulus set used by Kalish (1995). Thus, the present materials were better suited to capture differences in adults' conceptions of the two domains. Second, in the present study, the subjects were asked to rate either the typicality or the membership of items, whereas, in Kalish (1995), subjects were asked to judge both: first the typicality and then the membership. By not asking the subjects to make first typicality judgments in the present design, we avoided biasing their category membership judgments toward gradedness, as could have happened in Kalish (1995).

Altogether, the present data reveal a clear distinction in the nature of the relationship between typicality and category membership for animals and for artifacts. Although the linear regressions revealed that the data for animals did not fit an essentialist model perfectly-the slope was significantly different from zero-it seems to us these data are nonetheless better explained by an essentialist account with certain qualifications than by a similarity-based account.

According to an essentialist account, what matters most for determining the category membership of an exemplar in an essentialized domain is whether or not the exemplar is believed to have the essential features of a category. If it is believed to have such features, then the exemplar is considered a full member of the category. If it is believed not to have the essential properties, it is considered absolutely not a member of that category. It is evident from this formulation that for a person to make a definite categorization, the person has to have a clear belief about whether an exemplar has the essential properties of the category or not. If a person does not have a clear belief, then he or she has two options: (1) guess if the exemplar has the essential features of the category and categorize it accordingly or (2) be conservative and give a partial judgment of an exemplar's category membership. On the basis of the scatterplots of the data, our interpretation is that for animal items of high typicality (e.g., between 1 and 3), the subjects seemed quite certain that they had the essential properties of their assigned category and thus made mostly absolute positive judgments of category membership. For items of low typicality (e.g., in the upper $5 \mathrm{~s}$ and higher), the subjects seemed quite certain that they did not have the essential properties of their assigned category and thus predominantly made absolute negative judgments of category membership. Finally, the subjects seemed uncertain about whether or not items of intermediate degrees of typicality had the essential properties and, thus, averaging between "guessers" and "conservatives," yielded a pattern of graded ratings of membership. Some support for this interpretation comes from the distribution of responses for the animal items evident in Figure 1A. It is apparent that the variance in the responses was largest at intermediate degrees of typicality but was quite small at the extremes. In other words, at the extremes of typicality, the data fit quite well the idealized essentialist model of all-or-none categorization. However, further studies with more detailed analyses are needed to address this explanation.

Similarity-based models-specifically, exemplar models - arguably can explain a dissociation between judgments of typicality and judgments of category membership, such as the one evident for animals in the present study (Nosofsky, 1988). In general, though, similaritybased models assume that judgments of category membership are not all-or-none, except for exemplars very close to a prototype. In opposition to this conjecture, the data for animals reveal that the proportion of all-or-none judgments was quite similar for items with mean typicality ratings in the lower $1 \mathrm{~s}$ through items with mean typicality ratings in the upper $2 \mathrm{~s}$ and for items at the other end of the typicality spectrum.

Nonetheless, a similarity-based model—specifically, a prototype model--seems the best fit for the artifact data. As can be seen in Figure 1B, absolute categorization of artifact items dropped consistently with a decrease in the typicality of the items. For items of very low typicality, though, absolute categorization started to rise back up, primarily because of an increase in judgments of absolute negative categorization. Furthermore, the fact that the variance in the responses remained uniformly small across the entire range of typicality indicates that the relationship between typicality and cate- 
gory membership was constantly strong, as predicted by a prototype model (Hampton, 1995, 1998).

It is important to point out that the present study does not supply a definitive answer as to whether animals, but not artifacts, are essentialized. The goal of this study was to address the implications of this question with respect to one particular measure: the degree of absolute categorization. Further studies are still needed to assess domain differences in other respects (e.g., Braisby, Franks, \& Hampton, 1996). Nonetheless, as we argued earlier, this measure is of fundamental theoretical and empirical importance to the essentialist account. The finding that adults believe that membership in animal categories is more of an absolute matter than is membership in artifact categories adds supporting evidence for converging findings from anthropology (Atran, 1995), cognitive and developmental psychology (Gelman, Coley, \& Gottfried, 1994; Keil, 1995), and philosophy (Schwartz, 1978) that people's reasoning about animals is characterized by essentialist beliefs.

\section{REFERENCES}

Armstrong, S. L., Gleitman, L., \& Gleitman, H. (1983). What some concepts might not be. Cognition, 13, 263-308.

ATRAN, S. (1995). Causal constraints on categories and categorical constraints on biological reasoning across cultures. In D. Sperber, D. Premack, \& A. Premack (Eds.), Causal cognition: A multidisciplinary debate (pp. 205-233). Oxford: Oxford University Press.

BARR, R. A., \& CAPLAN, L. J. (1987). Category representations and their implications for category structure. Memory \& Cognition, 15, 397-418.

Battig, W. F., \& Montague, W. E. (1969). Category norms for verbal items in 56 categories. Journal of Experimental Psychology Monograph, 80(3, Pt. 2).

Braisby, N., Franks, B., \& Hampton, J. (1996). Essentialism, word use, and concepts. Cognition, 59, 247-274.

Diesendruck, G., Gelman, S. A., \& Lebowitz, K. (1998). Conceptua and linguistic biases in children's word learning. Developmental Psychology, 34, 823-839.

Gelman, S. A. (1988). The development of induction within natural kind and artifact categories. Cognitive Psychology, 20, 65-95.
Gelman, S. A., Coley, J. D., \& Gott fried, G. M. (1994). Essentialist beliefs in children: The acquisition of concepts and theories. In L. A Hirschfeld \& S. A. Gelman (Eds.), Mapping the mind: Domain specificity in cognition and culture (pp. 341-365). Cambridge: Cambridge University Press.

Gelman, S. A., \& Markman, E. M. (1986). Categories and induction in young children. Cognition, 23, 183-209.

HAMPTON, J. A. (1995). Testing the prototype theory of concepts. Jour nal of Memory \& Language, 34, 686-708.

Hampton, J. A. (1998). Similarity-based categorization and fuzziness of natural categories. Cognition, 65, 137-165.

KALISH, C. W. (1995). Essentialism and graded membership in animal and artifact categories. Memory \& Cognition, 23, 335-353.

KALISH, C. W. (1998). Natural and artifactual kinds: Are children realists or relativists about categories? Development Psychology, 34, 376-391.

KEIL, F. C. (1989). Concepts, kinds, and cognitive development. Cambridge, MA: MIT Press.

KEIL, F. C. (1995). The growth of causal understanding of natural kinds In D. Sperber, D. Premack, \& A. Premack (Eds.), Causal cognition: A multidisciplinary debate (pp. 234-262). Oxford: Oxford University Press.

MALT, B. C. (1990). Features and beliefs in the mental representation of categories. Journal of Memory \& Language, 29, 289-315.

Malt, B. C., \& Johnson, E. C. (1992). Do artifact concepts have cores? Journal of Memory \& Language, 31, 195-217.

MCCloskey, M. E., \& GlucksberG, S. (1978). Natural categories: Well defined or fuzzy sets? Memory \& Cognition, 6, 462-472.

MEdin, D. L. (1989). Concepts and conceptual structure. American Psychologist, 44, 1469-1481.

MURPHY, G. L., \& MEDIN, D. L. (1985). The role of theories in conceptual coherence. Psychological Review, 92, 289-316.

NosofSKy, R. M. (1988). Exemplar-based accounts of relations between classification, recognition, and typicality. Journal of Experimental Psychology: Learning, Memory, \& Cognition, 14, 700-708.

RIPS, L. J. (1989). Similarity, typicality, and categorization. In S. Vosniadou \& A. Ortony (Eds.), Similarity and analogical reasoning (pp. 21-59). New York: Cambridge University Press.

Rosch, E. (1975). Cognitive representations of semantic categories. Journal of Experimental Psychology: General, 104, 192-233.

Rosch, E. (1978). Principles of categorization. In E. Rosch \& B. B. Lloyd (Eds.), Cognition and categorization (pp. 27-48). Hillsdale, $\mathrm{NJ}$ : Erlbaum.

Schwartz, S. P. (1978). Putnam on artifacts. Philosophical Review, 87, 566-574.

Wierzbicka, A. (1984). Apples are not a "kind of fruit": The semantics of human categorization. American Ethnologist, 11, 313-328. 


\section{APPENDIX \\ Items and Categories Used in the Study}

Animals

Bird: robin, peacock, ostrich, warbler, penguin, bat, cardinal, pelican, pterodactyl, flying squirrel, sparrow, turkey, chickadee, blue jay, goose, crow, pheasant, swan, flamingo, emu, eagle, dragonfly

Dog: German shepherd, basset hound, dachshund, St. Bernard, Pekinese, Chihuahua, collie, pinscher, beagle, Newfoundland, wolf, retriever, fox, terrier, coyote, schnauzer, hyena, husky, chow chow, cocker spaniel

Fish: tuna, whale, dolphin, mackerel, halibut, eel, salmon, shrimp, snapper, squid, piranha, sturgeon, shark, tadpole, crab, trout, grouper, haddock, sardine, walleye, octopus, bass

Insect: mosquito, mouse, locust, caterpillar, virus, slug, butterfly, beetle, louse, germ, bacteria, cockroach, worm, termite, centipede, snail, dragonfly, spider, fly, praying mantis, ant, bee, cricket

Mammal: cat, panther, bison, hog, elk, beaver, bear, turtle, llama, alligator, mink, skunk, cow, lion, lizard, frog, chipmunk, antelope, gazelle, jaguar, dog, horse

Reptile: snake, spider, chameleon, crocodile, lizard, iguana, eel, worm, turtle, cobra, coral, salamander, tortoise, frog, python, alligator

\section{Artifacts}

Car: coupe, scooter, hatchback, RV, roadster, van, limousine, tricycle, pickup, buggy, trailer, station wagon, truck, convertible, tractor, sedan, dragster, jeep, bus, trolley

Chair: rocker, love seat, stool, recliner, stoop, bean bag, couch, tree stump, ottoman, sofa, bench, mat, perch, throne, hammock, swing

Clothing: pants, Bermudas, girdle, watch, vest, purse, jumper, robe, suit, shirt, apron, nylons, bathing suit, rings, boots, earmuffs, socks, skirt, cap, necklace, shoes

Furniture: table, bar, picture, stove, vase, refrigerator, cushion, stereo, mirror, ashtray, bookcase, bench, cupboard, bed, chair, drawers, clock, rocker, telephone, sofa, piano, desk, lamp

Shoe: slippers, roller blades, heels, sandals, boots, sneakers, tennis, flippers, oxfords, socks, cleats, galoshes, skates, loafers, pumps, skis, moccasins, clogs, flip-flops

Tool: hammer, answering machine, saw, yardstick, hanger, wedge, crane, glue, ax, hatchet, wood, bench, ladder, screwdriver, spoon, paper clip, drill, vise, knife, nails, bolts, tape, cement

Vehicle: airplane, tornado, tank, train, feet, surfboard, roller blades, carriage, car, bus, escalator, rocket, truck, elevator, elephant, river, helicopter, horse, camel, boat, skateboard, submarine, subway, sled

Weapon: gun, ice pick, pipe, rock, bow, slingshot, lance, hand, dagger, revolver, hatchet, knife, gas, rifle, scissors, words, foot, glass, bomb, rope, shoes, sword 